\title{
Proton beam radiotherapy (PBR) for the treatment of retinal capillary haemangioblastoma stabilises tumour progression but with poor visual outcomes
}

\author{
Rumana N. Hussain ${ }^{1} \cdot$ Syed Hassan ${ }^{1} \cdot$ V. Ho ${ }^{1} \cdot{\text { Andrzej Kacperek } \mathbb{D}^{1} \cdot \text { Douglas Errington }^{1} \cdot \text { Heinrich Heimann }}^{1}$
}

Received: 12 June 2018 / Revised: 22 January 2019 / Accepted: 6 February 2019 / Published online: 21 February 2019

(c) The Royal College of Ophthalmologists 2019

Retinal capillary haemangioblastomas $(\mathrm{RCH})$ may occur sporadically or as part of von Hippel-Lindau disease (VHL), an autosomal dominant condition. Mutations in the VHL gene, a tumour suppressor gene, cause abnormal production of the VHL protein and the subsequent uncontrollable cellular division results in the development of vascular tumours and cysts in the central nervous system and elsewhere. Approximately $2-4 \%$ of intraocular haemangioblastomas are juxtapapillary where the risk of visual loss is higher [1] and the destructive effects of laser photocoagulation, cryotherapy and brachytherapy usually lead to severe and permanent visual loss.

We report our experience with proton beam radiotherapy (PBR) of RCH located at the optic disc or unresponsive to other treatments. Four daily fractions were administered, with a total dose of 18 proton Gy.

Eight patients (three male, five female) were treated with mean age 49 years (range, 29-84 yrs). Four patients had a genetic diagnosis of VHL. All patients had juxtapapillary haemangioblastomas and two also had mid peripherallesions.

Basal tumour diameter at presentation was $3.1-7.2 \mathrm{~mm}$ (mean $5.0 \mathrm{~mm}$ ) with thickness of 1.2-4.2 mm (mean $2.4 \mathrm{~mm}$ ). Visual acuity ranged from $0.0 \log$ MAR to hand movements (mean 0.9). All patients had exudative changes. Six patients had previous unsuccessful therapy: argon laser photocoagulation $(n=3)$, photodynamic therapy $(n=4)$, intravitreal anti-VEGF injections (bevacizumab or abflibercept) $(n=4)$, ruthenium-106 plaque brachytherapy $(n=2)$ and vitrectomy with endolaser $(n=1)$ (Table 1$)$.

Rumana N. Hussain

Rumana.hussain@rlbuht.nhs.uk

1 Liverpool Ocular Oncology Service, St Paul's Eye Unit, Royal Liverpool University Hospital, Liverpool L7 8XP, UK
All patients were followed up for 3-7 years. Within three months of treatment, all haemangioblastomas regressed (mean longitudinal measurements $3.2 \mathrm{~mm}$, depth $1.8 \mathrm{~mm}$ ) with a decrease in tumour volume (decrease $1.6 \mathrm{~mm}$ longitudinally and $1.2 \mathrm{~mm}$ depth), continuing over 3 years. All lesions demonstrated resolution of subretinal fluid within 3-6 months; however three patients developed radiation-induced maculopathy between 12 and 22 months and were treated with intravitreal bevacizumab. Vision was stabilised in all cases but the majority had very poor vision at baseline and no improvement following therapy (baseline logMAR 0.0-Hand motions; last follow up 0.0-Perception of light; Table 1).

Treatment of juxtapapillary capillary haemangioblastomas is challenging. External beam radiotherapy (EBR) [2] has been successful but increases the risk of secondary central nervous system malignancies [3], as well as the potential development of radiation effects to both the retina and the optic nerve. Proton beam therapy has been described in two small series utilising $20 \mathrm{~Gy}$ but $3 / 8$ still developed optic neuropathy or maculopathy $[4,5]$.

PBR may be considered in patients where other treatment modalities fail to control aggressive disease in patients with juxtapapillary or peri-macular lesions. This may help with control of eye threatening exudative complications. Although the treatment enables control of tumour progression and growth, the high rate of radiation maculopathy despite reduced radiation doses is disappointing. As such this treatment should be considered only as a stabilising strategy as visual acuity does not improve; laser therapy is an alternative strategy, however in the peri-papillary region will certainly result in visual field defects. 


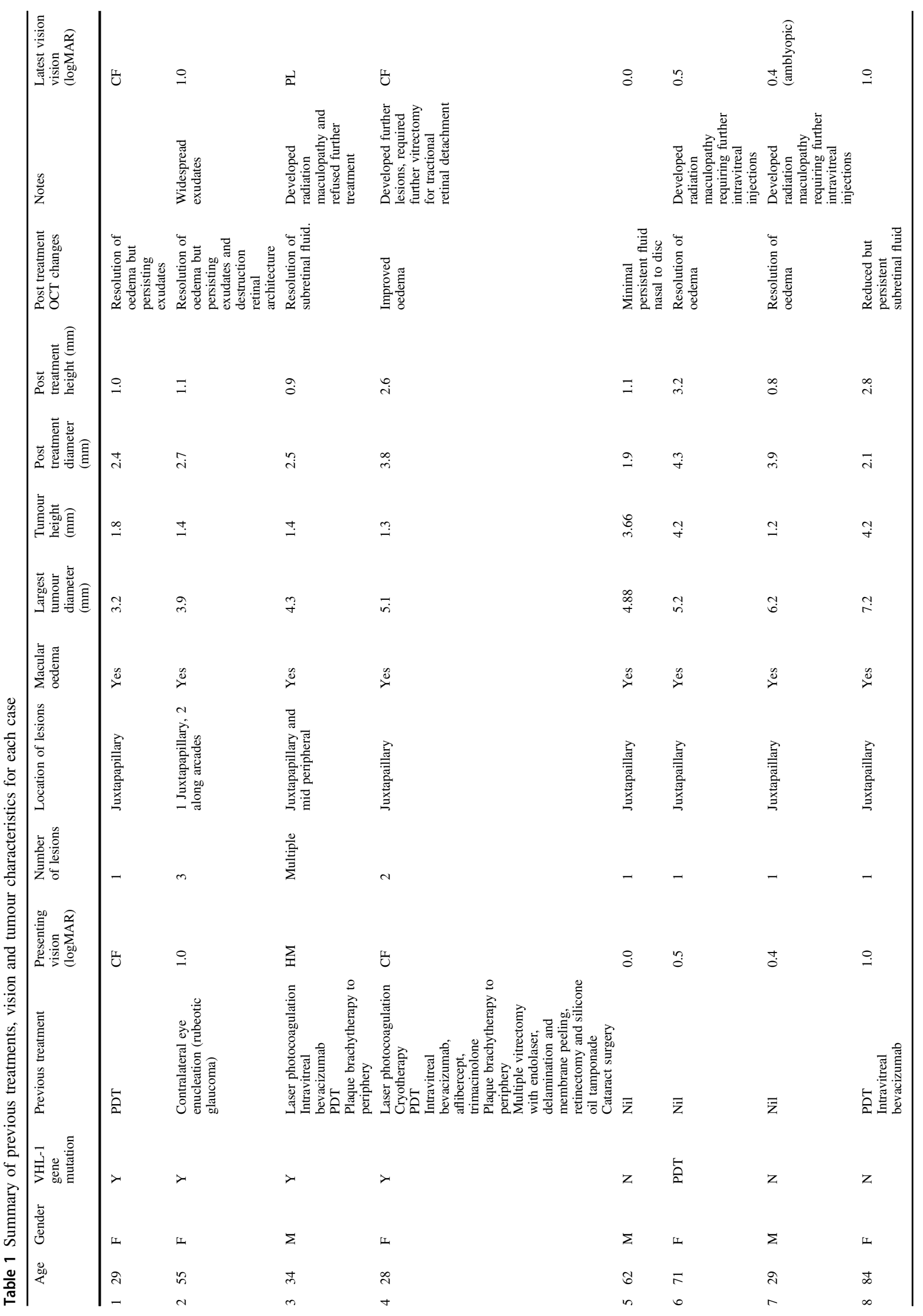




\section{Compliance with ethical standards}

Conflict of interest The authors declare that they have no conflict of interest.

Publisher's note: Springer Nature remains neutral with regard to jurisdictional claims in published maps and institutional affiliations.

\section{References}

1. Wong WT, Agron E, Coleman HR, Tran T, Reed GF, Csaky K, et al. Clinical characterization of retinal capillary hemangioblastomas in a large population of patients with von Hippel-Lindau disease. Ophthalmology. 2008;115:181-8.

2. Raja D, Benz MS, Murray TG, Escalona-Benz EM, Markoe A. Salvage external beam radiotherapy of retinal capillary hemangiomas secondary to von Hippel-Lindau disease: visual and anatomic outcomes. Ophthalmology. 2004;111:150-3.

3. Forrest AW. Tumour following radiation about the eye. Trans Am Acad Ophthalmol Otolaryngol. 1961;65:694-717.

4. Palmer JD, Gragoudas ES. Advances in treatment of retinal angiomas. Int Ophthalmol Clin. 1997;37:159-70.

5. Seibel I, Cordini D, Hager A, Riechardt A, Klein J, Heufelder J, et al. Long-Term Results After Proton Beam Therapy for Retinal Papillary Capillary Hemangioma. Am J Ophthalmol. 2014; 158:381-6.

\title{
Comment on: Clinical outcomes in Caucasian patients with polypoidal choroidal vasculopathy
}

\author{
H. D. Jeffry Hogg $\mathbb{D}^{1,2} \cdot$ S. James Talks ${ }^{2}$
}

Received: 25 January 2019 / Accepted: 31 January 2019 / Published online: 4 March 2019

(c) The Royal College of Ophthalmologists 2019

We have read the recent article from Agorogiannis et al. [1] reporting real world data from the treatment of polypoidal choroidal vasculopathy (PCV) in a Caucasian cohort with great interest. As described, there is a paucity of data from Caucasian individuals to inform the management of PCV, and so we aim to augment their informative dataset with longer follow-up and a greater representation of verteporfin photodynamic therapy (PDT) (Table 1).

Mean (SD) gain in ETDRS letters at one, two, three and four years was 0.8 (17.2), -1.6 (21.7), -1.6 (24.1) and -8.5 (29.7), respectively. Central foveal thickness (CFT) also improved at those timepoints by $68.0 \mu \mathrm{m}$ (204.9), 96.7 $\mu \mathrm{m}$ (124.9), $83.5 \mu \mathrm{m}$ (153.2) and $128.6 \mu \mathrm{m}$ (142.6). Although the mean acuity gained from PDT monotherapy was greater than from the combination of PDT and antivascular endothelial growth factor (anti-VEGF) treatment at

\footnotetext{
H. D. Jeffry Hogg

Jeffry.hogg@ncl.ac.uk

1 The University of Newcastle upon Tyne, Newcastle upon Tyne, Tyne and Wear NE1 7RU, UK

2 The Newcastle upon Tyne Hospitals NHS Foundation Trust, Newcastle upon Tyne, Tyne and Wear NE1 7RU, UK
}

Table 1 Demographics and descriptors of 29 eyes from Caucasian patients undergoing treatment for polypoidal choroidal vasculopathy who were subsequently followed up for 6 months or more

\begin{tabular}{lll}
\hline Gender & Female (\%) & $18(62.1)$ \\
Laterality & Male (\%) & $11(37.9)$ \\
& Left (\%) & $15(51.7)$ \\
Location & Right (\%) & $14(48.3)$ \\
& Macular (\%) & $4(13.8)$ \\
& Peripapillary (\%) & $22(75.9)$ \\
Follow-up from treatment & Peripheral (\%) & $3(10.3)$ \\
initiation (months) & $\%$ at 1 year & 86.2 \\
& $\%$ at 2 years & 72.4 \\
Final follow-up status & $\%$ at 3 years & 51.7 \\
& $\%$ at 4 years & 31 \\
& Under follow-up (\%) & $21(72.4)$ \\
& Discharged (\%) & $6(20.7)$ \\
Treatment received & Lost to follow-up (\%) & $1(3.4)$ \\
& Deceased (\%) & $1(3.4)$ \\
Initial central foveal & Anti-VEGF IVIs (\%) & $3(10.3)$ \\
thickness & PDT (\%) & $12(41.4)$ \\
\hline & Both (\%) & $14(48.3)$ \\
& Mean ETDRS letters & $66.7(14.3)$ \\
& (SD) & $332.3(68)$ \\
\hline
\end{tabular}

$S D$ standard deviation, $V E G F$ vascular endothelial growth factor, $P D T$ photodynamic therapy, IVI intravitreal injection. 